\title{
A Survey on Classification of ECG Signal Study
}

\author{
Taha E. Taha \\ Electronics \& Comm. Eng. Dept., \\ Faculty of Electronic Eng., \\ Menoufia University, Menouf \\ 32952, Egypt
}

\author{
Ayman El-Sayed \\ Computer Science \& Eng. Dept., \\ Faculty of Electronic Eng., \\ Menoufia University, Menouf \\ 32952, Egypt
}

\author{
Salma R. El-Soudy \\ Computer Science \& Eng. Dept., \\ Faculty of Electronic Eng., \\ Menoufia University, Menouf \\ 32952, Egypt
}

\begin{abstract}
Electrocardiogram (ECG) is a non-linear dynamic signal which plays the main role in diagnosis heart diseases. Classification of ECG signal is one of the most important reason of diagnosing the heart diseases. Detecting accurate ECG signal not only the most difficult task but also classifying heart signal is very difficult task. There are many types of classifiers are available for ECG classification. The most popular classifier that used in ECG classification is Artificial Neural Network (ANN) and in second degree is Support Vector Machine (SVM). In this paper, we discuss a survey of preprocessing, ECG database, feature extraction and classifiers. This paper also discusses background of Electrocardiogram, evaluation matrices of classifiers and issues of classifiers.
\end{abstract}

\section{Keywords}

Electrocardiogram, ECG classification, preprocessing, ANN, SVM, feature extraction, MIT-BIH database, Pan-Tompkins

\section{INTRODUCTION}

ECG is a medical signal which test the abnormality of cardiac by measuring the electrical activity of the heart. ECG signal is a tiny electrical impulse spread through the heart muscle. ECG signals can be detected by ECG machine which records the electrical activity of the heart and displays this data as signal on a paper sheet. ECG helps physician to diagnose the sources of the chest pain and heart diseases.

ECG has a standard shape for a normal heart. Any change or damage in the heart rhythm can be reflected on the shape of ECG sheet. Physicians check ECG sheet for the patients to ensure if there is a heart disease due to smoking, family history, high cholesterol, or high blood pressure. ECG is used to detect heart disorders including abnormal heart rhythms, heart attack and other diseases. Early and accurate discovery of ECG arrhythmia helps physicians to distinguish between different heart infections. The abnormality of the ECG signal can be identified by using classification. Accurate classification helps the physician to find the correct treatment therapy for the patient [1].

The general definition of classification is a general process related to categorization, the process in which ideas and objects are recognized, differentiated, and understood. The mathematical definition of classification is identifying of a set of categories which belongs to a new observation based on a training set of data. Classification of ECG is a hard task because of many issues involved classification process[2].

ECG classification steps include preprocessing, feature extraction, feature normalization and classification. In this paper, a detailed survey is presented about different classifiers and different performance measurements.
In order to evaluate the performance of ECG classification techniques, there are evaluation metrics such as accuracy, sensitivity, specificity and positive predictivity [3]. Accuracy is defined as the ratio of the number of correctly classified patterns to the total number of patterns classified. Sensitivity is defined as the ratio of correct events among all events. Specificity is defined as the ratio of correctly classified nonevents among all nonevents. Positive predictivity is defined as the ratio of the rate of correctly classified events in all detected events.

The rest of this paper is organized as follows. Section 2 presents brief background knowledge about Electrocardiogram (ECG). Section 3 describes issues in ECG classification. Section 4 explain taxonomy feature extraction techniques. Section 5 discuss taxonomy ECG classification techniques. Section 6 ECG Database Finally, the conclusion is presented in section 7 .

\section{BACKGROUND KNOWLEDGE}

In ECG, the magnitude and direction of heart activity generated by depolarization and repolarization of atria and ventricles are recorded. Figure.1 describes ECG signal and different intervals P-R interval, ST interval and QRS complex interval. ECG consists of five peaks and valleys labeled by the letters $\mathrm{P}, \mathrm{Q}, \mathrm{R}, \mathrm{S}$ and $\mathrm{T}$ [4]. The most dominant feature of them is QRS complex.

The signal is generated as the vena cava fill the right atrium of the heart with blood from other parts of the body. The signal spreads across the cells of the heart right and left atria. This signal causes the atria to contract. This action pushes blood through the open valves from the atria into both ventricles. The signal arrives at the AV node near the ventricles. It slows for an instant to allow the heart's right and left ventricles to fill with blood. The signal is released and moves along a pathway called the bundle of His, which is located in the walls of the heart's ventricles. From the bundle of His, the signal fibers divide into left and right bundle branches through the Purkinje fibers. These fibers connect directly to the cells in the walls of the heart's left and right ventricles.

The signal spreads across the cells of ventricle walls, and both ventricles contract. However, this doesn't happen at exactly the same moment. The left ventricle contracts an instant before the right ventricle. This pushes blood through the pulmonary valve (for the right ventricle) to lungs, and through the aortic valve (for the left ventricle) to the rest of body. As the signal passes, the walls of the ventricles relax and await the next signal. This process continues over and over as the atria refill with blood and more electrical signals come from the SA node figure 2 shows the electrical system of ECG[5]. 


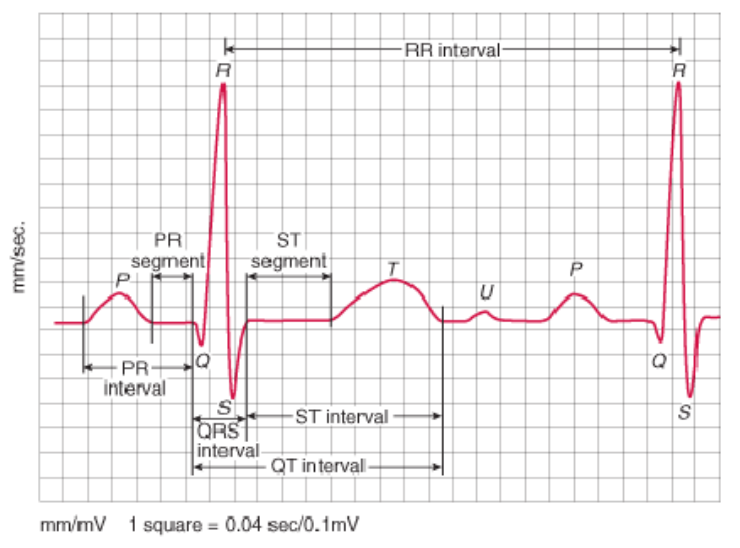

Fig 1: ECG Waveform and QRS complex interval [4]

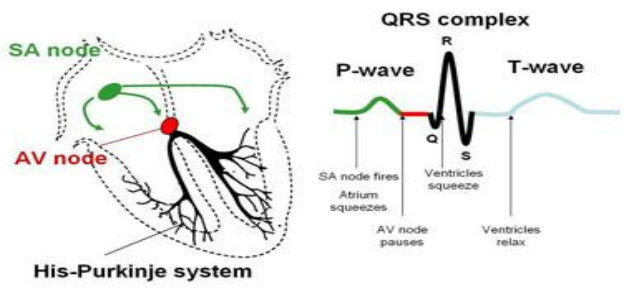

Fig 2: ECG signal electrical system [6]

According to QRS-Complex, the Q wave is the introductory descending diversion; typical $\mathrm{Q}$ waves last $<0.05 \mathrm{sec}$ in all leads with the exception of V1-3, in which any Q wave is viewed as strange, demonstrating past or current dead tissue. The $\mathrm{R}$ wave is the primary upward diversion; criteria for ordinary tallness or size are not total, but rather taller $\mathrm{R}$ waves might be brought on by ventricular hypertrophy. A second upward diversion in a QRS complex is assigned $\mathrm{R}^{\prime}$. The $\mathrm{S}$ wave is the second descending redirection if there is a $\mathrm{Q}$ wave and the principal descending avoidance if not. The QRS complex might be R alone, QS (no R), QR (no S), RS (no Q), or RSR', contingent upon the ECG lead, vector, and vicinity of heart issue. By and large QRS complex is utilized to recognize arrhythmias and distinguish the issues of strange ECG signals. The change in QRS complex depends on time and debased with clamor. QRS complex is unforgiving in light of the fact that distinguishing R-wave is confused as Rwave is the most noteworthy purpose of the QRS. There are other edges in distinguishing QRS complex such $\mathrm{P}$ and $\mathrm{T}$ waves have comparable qualities as QRS. The other test in distinguishing QRS complex is the clamor where there are numerous wellsprings of commotion, for example, patient condition, baseline wander, patient movement, input power supply and weak contact of ECG electrodes, de-noising the sign is fundamental for QRS detector. The QRS-complex detection in ECG is a difficult problem to identify because it is a subject to physiological variations due to patient and different types of noise [7]. Classification of ECG is very important in diagnosing heart cases.
Table 1. ECG features and normal duration [8]

\begin{tabular}{|c|c|c|}
\hline Feature & Description & Duration \\
\hline $\begin{array}{l}\mathrm{RR} \\
\text { interval }\end{array}$ & $\begin{array}{l}\text { The interval between an } R \\
\text { wave and the next } R \text { wave, } \\
\text { normal resting heart rate is } \\
\text { between } 60 \text { and } 100 \mathrm{bpm}\end{array}$ & $0.6-1.2 \mathrm{~s}$ \\
\hline $\mathrm{P}$ wave & $\begin{array}{l}\text { During normal atrial } \\
\text { depolarization, the main } \\
\text { electrical vector is directed } \\
\text { from the SA node towards the } \\
\text { AV node, and spreads from } \\
\text { the right atrium to the left } \\
\text { atrium }\end{array}$ & $80 \mathrm{~ms}$ \\
\hline $\begin{array}{l}\mathrm{PR} \\
\text { interval }\end{array}$ & $\begin{array}{l}\text { The PR interval is measured } \\
\text { from the beginning of the } \mathrm{P} \\
\text { wave to the beginning of the } \\
\text { QRS complex }\end{array}$ & $\begin{array}{c}120- \\
200 \mathrm{~ms}\end{array}$ \\
\hline $\begin{array}{l}\text { PR } \\
\text { segment }\end{array}$ & $\begin{array}{l}\text { measured from the beginning } \\
\text { of the } \mathrm{P} \text { wave to the beginning } \\
\text { of the QRS complex }\end{array}$ & $50-120 \mathrm{~ms}$ \\
\hline $\begin{array}{l}\text { QRS } \\
\text { Complex }\end{array}$ & $\begin{array}{l}\text { The QRS complex reflects the } \\
\text { rapid depolarization of the } \\
\text { right and left ventricles. They } \\
\text { have a large muscle mass } \\
\text { compared to the atria }\end{array}$ & $80-120 \mathrm{~ms}$ \\
\hline J-point & $\begin{array}{l}\text { The point at which the QRS } \\
\text { complex finishes and the ST } \\
\text { segment begins, it is used to } \\
\text { measure the degree of ST } \\
\text { elevation or depression } \\
\text { present }\end{array}$ & N/A \\
\hline $\begin{array}{l}\text { ST } \\
\text { segment }\end{array}$ & $\begin{array}{l}\text { The ST segment connects the } \\
\text { QRS complex and the T wave. } \\
\text { The ST segment represents } \\
\text { the period when the ventricles } \\
\text { are depolarized }\end{array}$ & $80-120 \mathrm{~ms}$ \\
\hline $\begin{array}{l}\text { ST } \\
\text { interval }\end{array}$ & $\begin{array}{l}\text { The ST interval is measured } \\
\text { from the } J \text { point to the end of } \\
\text { the } T \text { wave }\end{array}$ & $320 \mathrm{~ms}$ \\
\hline $\mathrm{T}$ wave & $\begin{array}{l}\text { The } \mathrm{T} \text { wave represents the } \\
\text { repolarization (or recovery) of } \\
\text { the ventricles. The interval } \\
\text { from the beginning of the } \\
\mathrm{QRS} \text { complex to the apex of } \\
\text { the } \mathrm{T} \text { wave is referred to as } \\
\text { the absolute refractory period. } \\
\text { The last half of the } \mathrm{T} \text { wave is } \\
\text { referred to as the relative } \\
\text { refractory period (or } \\
\text { vulnerable period). }\end{array}$ & $160 \mathrm{~ms}$ \\
\hline QT & $\begin{array}{l}\text { Measured from the beginning } \\
\text { of QRS complex to the end } \mathrm{T} \\
\text { wave }\end{array}$ & $420 \mathrm{~ms}$ \\
\hline U & $\begin{array}{l}\text { Normally has low amplitude } \\
\text { and often it is completely } \\
\text { absent }\end{array}$ & $\begin{array}{l}\text { Not } \\
\text { mentioned }\end{array}$ \\
\hline
\end{tabular}

The classification of ECG has four steps; preprocessing, feature extraction, normalization, and classification. In preprocessing step, ECG signals may contain several types of noise like white noise and baseline wander noise. In this step the main target is removing noise which can effect on features used in classification. In feature extraction step, ECG features used as inputs to classifier are extracted from the signal. 
Researchers use many techniques for extracting ECG features like Discrete Wavelet Transform (DWT), Continuous Wavelet Transform (CWT), Discrete Cosine Transform (DCT), STransform (ST), Discrete Fourier transform (DFT), Principal Component Analysis (PCA), Daubechies wavelet (Db4), PanTompkins algorithm and Independent Component Analysis (ICA). According

to normalization of features, Z-score, and Unity Standard Deviation (SD) are used.

The problem of ECG classification which faces the researchers is the multi-class classification problem that contains many classes like Normal, RBBB (Right Bundle Branch Block), LBBB (Left Bundle Branch Block), suprventrical and Atrial escape beat. ECG signal can be classified with two ways; classifying as a signal and classifying as individual beats. Each beat consists of P, QRS, $\mathrm{T}$ and $\mathrm{U}$ wave. Each signal consists of thousands of beats. The techniques used for classification are Multilayer Perceptron Neural Network (MLPNN), Fuzzy C-Means clustering (FCM), Feed forward neuron-fuzzy, ID3 decision tree, Support Vector Machine (SVM), Quantum Neural Network (QNN), Radial Basis Function Neural Network (RBFNN), Type2 Fuzzy Clustering Neural Network (T2FCNN) and Probabilistic Neural Network (PNN) classifier.

In this paper, Wavelet transform and Pan-Tompkins are chosen for preprocessing and ANN, SVM and MLPNN are chosen for classification.

\section{ISSUES IN ECG CLASSIFICATION}

There are many issues in classification of ECG signals, these issues are:

(a) Absent of standardization of ECG features where the accuracy of feature extraction method depends on the selected features. Therefore, any small error in these features causes misclassification over all data sets.

(b) Selection of suitable classifier is one of the issues because the accuracy of classifier depends on more than one parameter such as arrhythmia type, selected feature extraction method and arrhythmia database.

(c) No rules for ECG classification.

(d) Uniqueness of the ECG pattern where the individuality of the ECG pattern refers to the probability of intra-class similitude and interclass variability of testing examples seen in ECG data.

(e) Variability of the ECG features where heart rate of person is changing according to physiological and mental conditions. Stress, energy, exercise, and other working exercises may change the heart rate. The variation of RR interval, PR interval, QT interval are the main cause of changing the heart rate. These features should be precisely changed and the effect of the fluctuating heart rate should be dispensed with.

(f) Each patient has different ECG waveform where ECG signal verify from one patient to anther as may have different slops of amplitude, time, signal which make changes in ECG patterns, so that the classification process need to classify signals carefully.

\section{TAXONOMY IN FEATURE EXTRACTION}

The main techniques used for feature extraction are wavelet transformation, discrete wavelet transform (DWT), and Continuous wavelet transform (CWT) and Pan Tompkins.

\subsection{Wavelet Transform (WT)}

Principles- The general strategy of implementing the WT is passing the sign at the same time through specially designed filter high pass filter and low pass filter.[9]. The interesting points of wavelets are wavelet localization in both time and frequency, analyzing non-stationary signals such as ECG which have jumps and non-smooth features and separating a signal into multi-resolution components. In recent years, wavelet transform is suitable for representing ECG signals for identifying QRS complex features.

\subsubsection{Discrete wavelet transform (DWT)}

DWT is one of the most important applications of ECG feature extraction to compute and manipulate the data in compressed parameters. DWT has become a powerful technique in biomedical signal processing. DWT decomposes a signal at different scales. Using DWT gives the ability to obtain good separation of the QRS complexes from other ECG components and noise in the time-scale plane. DWT is a method of reduce noise and detect R-peak of ECG signal. However, it does not work as expected in the detection and delineation of low frequency waves such as $\mathrm{P}$ and $\mathrm{T}[10]$. The discrete wavelet transform (DWT) can be writing as the following:

Equation (1) represents the discrete wavelet transform (DWT).

$$
T_{m, n}=\int_{-\infty}^{\infty} x(t) \psi_{m, n}(t) d t
$$

Where $\mathrm{m}$ and $\mathrm{n}$ are signal scale and location. The approximation coefficient of the signal is shown in equation (2).

$$
S_{m, n}=\int_{-\infty}^{\infty} x(t) \phi_{m, n}(t) d t
$$

According to limited length of discrete signal, equation (3) represents a discrete guess of the signal.

$$
X_{0}=X_{M(t)} \sum_{m=1}^{M} d m(t)
$$

The mean signal approximation at scale $\mathrm{m}$ is shown in equation (4)

$$
\begin{aligned}
& X_{M(t)} \\
& =S_{m, n} \phi_{M, n}(t)
\end{aligned}
$$

Equation (5) represents the detail signal approximation corresponding to scale $\mathrm{m}$, for finite length signal.

$$
d m(t)=\sum_{n=0}^{M-m} T_{m, n} \psi_{m, n}
$$

The signal approximation at a specific scale is a combination of the approximation and detail at the next lower scale:

$$
X_{m}(t)=X_{m-1}(t)-d_{m}(t)
$$


In multi-determination wavelet change, the first signal is connected through a high pass and a low pass filter what's more, gotten approximated and detail coefficients of the signal. The points of interest are the low-scale, high-frequency segments and approximations are the high-scale, low frequency parts of the signal[11] [12].

\subsubsection{Continuous wavelet transform (CWT)}

CWT is one of the most popular types of wavelet. CWT-based algorithm affords high time-frequency resolution which provides a better definition of the QRS modulus maxima curves. CWT converts a continuous signal into highly redundant signal of two continuous variables which are translation and scale. The resulting converted signal is easy to interpret and valuable for time - frequency analysis. CWTbased algorithm affords high time-frequency resolution which provides a better definition of the QRS modulus maxima curves. This allows them to follow QRS wave across scales in noisy signals, and better to define the spectral region corresponding to the QRS maxima peak [13].

Discussion- The wavelet is the most popular technique in preprocessing and feature extraction stage because of removing noise from ECG signal. There are many types of wavelet such as discrete wavelet (DWT) and continuous wavelet (CWT).

\subsection{Pan-Tompkins Algorithm}

Principles- Pan-Tompkins is one of the most popular algorithms included in virtually all biomedical signal processing textbooks. Pan and Tompkins (PT), also known as the low-pass differentiation algorithm (LPD), introduced a major evolution in ECG signal processing in 1985[14]. This basic algorithm covers 5 steps namely band pass filtering, differentiation, squaring, moving window integration, and thresholds adjustment

The first step is band pass filter which reduces the effect of many types of noise such as muscle noise, $60 \mathrm{HZ}$ interference, baseline wander and T-wave [14]. The target of band pass filter is maximizing the QRS energy to be $5-15 \mathrm{HZ}$. In this algorithm, the filter is implemented by cascaded high pass filter and low pass filter.

The second step is Differentiation where the QRS complex is found by differentiating the signal and getting the high slopes which normally identify the QRS from other components of ECG signal. The derivative procedure represses the low frequency component of $\mathrm{P}$ and $\mathrm{T}$ wave and provide the high frequency component arising from the high slops of QRScomplex.

After differentiation, the third step is squaring the signal. It means squaring point by point to make all points positive. The large differences resulting from QRS-complexes and small differences arising from $\mathrm{P}$ and $\mathrm{T}$ waves are repressed. The high frequency components in the signal are QRS-complexes.

The next step is moving window which preforms smoothing the output of preceding operation. The integrator sums the area under the squared waveform over suitable interval. Derivative operator finds the high slopes that normally distinguish the R peak from other ECG waves and suppresses the low frequency components of $\mathrm{P}$ and $\mathrm{T}$ waves. Squaring operation is point by point squaring of ECG signal. It is used for further enhancing high frequency components and suppressing the small differences arising from $\mathrm{P}$ and $\mathrm{T}$ waves.
Integration sums the area under the squared waveform over a suitable interval. It extracts the slope of the $\mathrm{R}$ wave. Signal to noise ratio increases after the ECG signal has passed from the band pass filter. Therefore, threshold adjustment is done and sensitivity of the algorithm is improved.

Discussion- Advantages of using Pan-Tomkins algorithm compared to other available techniques for feature extraction are sensitivity and efficiency of Pan-Tompkins algorithm are more than 99\% [15]. The computational efforts are also less than other algorithms. It includes noise removal and baseline wonder removal steps. Therefore, no need to use other techniques separately.

\section{TAXONOMY OF CLASSIFICATION TECHNIQUES}

There are many classifiers and classification techniques used to classify ECG signals such as Support Vector Machine (SVM), Artificial Neural Network (ANN) Multilayer Perceptron Neural Network (MLPNN).

\subsection{Support Vector Machine (SVM)}

Principles- SVM is a new paradigm of learning machine as it is a powerful, widely used technique for solving supervised classification problems due to it generalization ability. SVM has a regularization parameter, which makes the user think about avoiding over-fitting. SVM uses the kernel trick. Therefore, it can be built in expert knowledge about the problem via engineering the kernel. SVM is defined by a convex optimization problem (no local minima) for which there are efficient methods [16].

Discussion- The disadvantages of SVM are that the theory only really covers the determination of the parameters for a given value of the regularization and kernel parameters and choice of kernel. In a way, the SVM moves the problem of over-fitting from optimizing the parameters to model selection. Sadly kernel models can be quite sensitive to overfitting the model selection criterion [17].

\subsection{Artificial Neural Network (ANN)}

Principles- Artificial Neural Network (ANN) was used by many researchers for ECG classification. ANNs have many advantages such as data driven, self-adaptive, non-linear, fast, and accurate. It is also robust to noise and easily scalable. ANN gives non-straight mapping amongst inputs and output utilizing actuation capacity, for example, sigmoid to take care of non-straight issue, for example, grouping of ECG signals. It can accomplish comparative or preferable results over measurable or deterministic methodologies. Factual techniques perform useful for straight issues however it can't produce great results for non-straight issue because factual strategies are created considering the suspicion of given direct time arrangement. ANN can adaptively show the lower frequencies of the ECG which are characteristically nonstraight. ANN evacuates time varying and nonlinear noise characteristics of ECG signal [18].

Discussion- There are problems in using ANN. The main problem is that preparing calculation of ANN is not able to guarantee a worldwide least is achieved. It may not essentially give ideal answer for the whole 12-lead ECG grouping process. But it the most popular technique used in ECG classification with MIT-BIH database [1]. 


\subsection{Multilayer Perceptron Neural Network (MLPNN)}

Principles- This technique is used for ECG classification. This one is static and feed-forward in nature and has no delay or feedback loops. Precision of MLPNN increments with number of shrouded neurons. MLPNN performs static mapping, there are no inside progression [4][19].

Discussion- In MLPNN, once in a while over fitting issue may happen. To conquer issue of over fitting, we require early halting criteria. MLPNN gives some good results in beat classification.

\section{ECG DATABASE}

UCI Arrhythmia dataset[20] and MIT-BIH arrhythmia dataset (mitdb) are the most popular types of datasets that used by researchers. MIT-BIH database is most popular database used by researchers for ECG arrhythmia. MIT-BIH database contains 48 recording for half-hour extracts of two-channel wandering ECG recordings, acquired from 47 subjects contemplated by the BIH Arrhythmia Laboratory somewhere around 1975 and 1979. Twenty-three recordings were picked aimlessly from an arrangement of 4000 24-hour mobile ECG recordings gathered from a blended populace of inpatients (around 60\%) and outpatients (around 40\%) at Boston's Beth Israel Hospital; the remaining 25 recordings were chosen from the same set to incorporate less normal however clinically huge arrhythmias that would not be very much spoken to in a little irregular specimen The recordings were digitized at 360 examples for every second per channel with 11-bit determination over a $10 \mathrm{mV}$ range. Two or more cardiologists autonomously clarified every record; differences were made plans to acquire the PC clear reference explanations for every beat (roughly 110,000 comments taking all things together) included with the database. Approximated 109,000 beat labels are there in the database. The MIT-BIH ECG database contains three different files such as header file (.hea), a binary file (.dat) and a binary annotation file (.atr). Patient's history clinical information with detailed information such as number of tests, examining recurrence, organization of ECG signal, sort of ECG leads and number of ECG leads are portrayed in the header document. In parallel comment record, the signal is put away in 212 organization which implies every one specimen requires number of leads times 12 bits to be put away and the parallel comment record comprises of beat explanations[21] .

\section{CONCLUSION}

In this paper, the issues of classification, and the preprocessing techniques used to remove noise from ECG signals have been presented. Different types of feature extraction techniques, different types of classification techniques and performance metrics have been discussed. According to preprocessing and feature extraction techniques, wavelet transform and pan-Tompkins algorithms are the most popular techniques which are used. These ones ought to utilize calculation for pre-preparing what's more, feature extraction contrasted with wavelet technique because of extracting feature and removing noise utilizing wavelet one should utilize larger amount of decomposition. On the other hand, wavelet transform is more complex and need too much time to process. In classification stage, ANN and SVM have been used a lot. According to survey Neural Network gives good results for ECG classification and researchers prefer this method as it gives a good accuracy rate. The evaluation matrices for ECG classification are accuracy, sensitivity, and specificity to evaluate the performance of the classifier.

The future enhancement is to design an evaluation method which helps us to classify ECG with WEKA which helps to classify signal with more than one algorithm at same time.

\section{ACKNOWLEDGMENTS}

An acknowledgement to Academy of Scientific Research and Technology for its contributing constructively in completion of this paper.

\section{REFERENCES}

[1] S. H. Jambukia, "Classification of ECG signals using machine learning techniques : A survey," no. July, 2016.

[2] Y. N. Singh, S. K. Singh, and A. K. Ray, "Bioelectrical signals as emerging biometrics: Issues and challenges," ISRN Signal Process., vol. 2012, 2012.

[3] A. Gacek and W. Pedrycz, ECG Signal Processing, Classification and Interpretation: A Comprehensive Framework of Computational Intelligence. Springer London, 2011.

[4] V. S. R. Kumari and P. R. Kumar, "CARDIAC ARRHYTHMIA PREDICTION USING IMPROVED MULTILAYER PERCEPTRON NEURAL NETWORK," vol. 3, no. 4, pp. 73-80, 2013.

[5] "ECG waveform." [Online]. Available: https://www.nhlbi.nih.gov/health/healthtopics/topics/hhw/electrical. [Accessed: 01-Jul-2016].

[6] "ECG signal electrical system." [Online]. Available: http://www.lifebeatonline.com/en-US/explore-heartconditions/heart-smart.html. [Accessed: 01-Jun-2016].

[7] S. G. Hiremath, "WAVELET TRANSFORM BASED ON QRS DETECTION USING DIODIC ALGORITHM," pp. 909-915, 2014.

[8] "ECG features and normal duration." [Online]. Available: http://en.wikipedia.org/wiki/Electrocardiography. [Accessed: 01-Aug-2016].

[9] M. Sifuzzaman, M. R. Islam, and M. Z. Ali, "Application of Wavelet Transform and its Advantages Compared to Fourier Transform,” J. Phys. Sci., vol. 13, pp. 121-134, 2009.

[10] R. Raddadi and E. Abdelmounim, "Discrete Wavelet Transform Based Algorithm For Recognition Of QRS Complexes," 2014.

[11] S. C. Saxena, V. Kumar, and S. T. Hamde, "Feature extraction from ECG signals using wavelet transforms for disease diagnostics," Int. J. Syst. Sci., vol. 33, no. 13, pp. 1073-1085, 2002.

[12] R. J. Martis, U. R. Acharya, and L. C. Min, "ECG beat classification using PCA, LDA, ICA and Discrete Wavelet Transform," Biomed. Signal Process. Control, 2013.

[13] H. Nagendra, S. Mukherjee, and V. Kumar, “Application of Wavelet Techniques in ECG Signal Processing: An Overview," Int. J. Eng. Sci. Technol., vol. 3, no. 10, pp. 7432-7443, 2011. 
[14] J. Pan and W. J. Tompkins, "A real-time QRS detection algorithm.," IEEE Trans. Biomed. Eng., vol. 32, no. 3, pp. 230-236, 1985.

[15] S. L. Pingale, "Using Pan Tompkin' S Method, Ecg Signal Processing and Dignose Various Diseases in Matlab," no. 411052, pp. 57-61, 2014.

[16] S. S. Mehta and N. S. Lingayat, "Detection of QRS complexes in electrocardiogram using support vector machine.," J. Med. Eng. Technol., vol. 32, no. 3, pp. 206-215, 2008.

[17] G. C. Cawley and N. L. C. Talbot, "On over-fitting in model selection and subsequent selection bias in performance evaluation," J. Mach. Learn. Res., vol. 11, no. Jul, pp. 2079-2107, 2010.
[18] X. Q., H. Y.H., and T. W.J., "Neural-Network-Based Adaptive Matched Filtering for QRS Detection," IEEE Trans. Biomed. Eng., vol. 39, no. 4, pp. 317-329, 1992.

[19] M. Moavenian and H. Khorrami, "A qualitative comparison of artificial neural networks and support vector machines in ECG arrhythmias classification," Expert Syst. Appl., vol. 37, no. 4, pp. 3088-3093, 2010.

[20] "UCI." [Online]. Available: Available:https://archive.ics.uci.edu/ml/datasets/Arrhyth mia. [Accessed: 18-Dec-2016].

[21] "MIT-BIH Database." [Online]. Available: https://physionet.org/physiobank/database/mitdb/. [Accessed: 12-Jan-2015]. 